\title{
DESCOMPRESSÃO NEURAL ISOLADA OU ASSOCIADA À FUSÃO PÓSTERO-LATERAL NAS AFECÇÕES DEGENERATIVAS LOMBOSSACRAS: AVALIAÇÃO DA QUALIDADE DE VIDA E INCAPACIDADE FUNCIONAL PÓS-OPERATÓRIA
}

\author{
NEURAL DECOMPRESSION ALONE OR COMBINED WITH POSTEROLATERAL FUSION \\ IN LUMBOSACRAL DEGENERATIVE DISEASES: ASSESSMENT OF POSTOPERATIVE \\ QUALITY OF LIFE AND FUNCTIONAL DISABILITY \\ DESCOMPRESIÓN NEURAL AISLADA O COMBINADA CON LA FUSIÓN POSTEROLATERAL \\ EN LAS ENFERMEDADES DEGENERATIVAS LUMBOSACRAS: EVALUACIÓN DE LA \\ CALIDAD DE VIDA E INCAPACIDAD FUNCIONAL DESPUÉS DE LA OPERACIÓN
}

Alberto Ofenhejm Gotfryd', Douglas Romano Spolidoro², Patrícia Rios Poletto ${ }^{3}$

RESUMO

Objetivo: Comparar a qualidade de vida, a dor e a satisfação pessoal de pacientes submetidos à descompressão neural lombar isolada àqueles que tiveram a fusão póstero-lateral associada. Métodos: Participaram do estudo 44 indivíduos com diagnóstico de hérnia de disco e/ou estenose degenerativa central ou foraminal da coluna lombossacra tratados cirurgicamente. Os pacientes foram divididos em 2 grupos: "descompressão" (D) e "descompressão e fusão" (DF). O critério utilizado para definir a necessidade da artrodese foi a presença de deformidades ou instabilidade segmentar, mensurada através de radiografias simples e dinâmicas. Os pacientes preencheram questionários referentes ao acompanhamento pós-operatório (uso de medicamentos analgésicos e satisfação com o tratamento) e escala analógica visual de dor lombar e ciática. Além disto, foram aplicados os questionários Oswestry e SF-36 para avaliação da qualidade de vida. Resultados: Foram encontrados excelentes resultados no questionário Oswestry, bons níveis para os domínios "Dor" e "Capacidade Funcional" do SF-36, além de baixa intensidade de dor lombar e ciática em ambos os grupos analisados, não havendo diferenças estatisticamente significativas entre eles. Conclusões: Não encontramos diferenças em relação à qualidade de vida, à dor e à satisfação pessoal em pacientes submetidos à descompressão neural lombar isolada àqueles que tiveram a fusão associada, utilizando como critério indicativo para artrodese a presença de deformidades e/ou instabilidade segmentar.

Descritores: Coluna vertebral; Dor lombar; Artrodese, Descompressão cirúrgica; Qualidade de vida; Ciática; Discotomia.

\begin{abstract}
Objective: To compare the quality of life, pain and satisfaction of patients undergoing lumbar isolated decompression neural to those who had posterolateral fusion associated. Methods: The study enrolled 44 subjects with lumbar herniated discs and/or degenerative central or foraminal stenosis treated surgically. Patients were divided into two groups: "decompression" (D) and "decompression and fusion" (DF). The criteria used to define the need for arthrodesis was the presence of deformity or segmental instability, measured by plain and dynamic radiographs. The patients completed questionnaires regarding postoperative follow-up (use of analgesic medications and satisfaction with the treatment) and visual analog scale for lumbar pain and sciatica. Moreover, the questionnaires Oswestry and SF-36 were administered to assess quality of life. Results: We found excellent results in the Oswestry questionnaire, good levels for the domains "Pain" and "functional capacity" in the SF-36, and low intensity of back pain and sciatica in both groups analyzed, without statistically significant differences between them. Conclusions: We found no differences in the quality of life, pain and satisfaction in patients undergoing lumbar nerve decompression alone compared to those who had associated fusion, using as a criterion for arthrodesis the presence of deformities and/orsegmental instability.
\end{abstract}

Keywords: Spine; Low back pain; Arthrodesis; Surgical Decompression; Quality of life; Sciatica; Diskectomy.

\section{RESUMEN}

Objetivo: Comparar la calidad de vida, el dolor y la satisfacción personal de los pacientes sometidos a la descompresión neural aislada con aquellos que tuvieron la fusión posterolateral asociadas. Métodos: El estudio reclutó a 44 pacientes con hernias discales y/o estenosis degenerativa central o foraminal de la columna lumbosacra, tratados quirúrgicamente. Los pacientes fueron divididos en dos grupos: "descompresión" (D) y "descompresión y fusión" (DF). El criterio utilizado para definir la necesidad de artrodesis fue la presencia de deformidades o inestabilidad segmentaria, según lo medido por radiografías simples y dinámicas. Los pacientes completaron cuestionarios sobre seguimiento postoperatorio (uso de medicamentos analgésicos y satisfacción con el tratamiento) y la escala analógica visual para el dolor lumbar y ciática. Por otra parte, los cuestionarios Oswestry y SF-36 fueron aplicados para evaluar la calidad de vida. Resultados: Se encontraron excelentes resultados en el cuestionario de Oswestry, buenos niveles para los dominios de "dolor" y "capacidad funcional" en el SF-36, además de baja intensidad de dolor lumbar y ciática en los dos grupos analizados; no hubo diferencias estadísticamente significativas entre ellos. Conclusiones: No se encontraron diferencias en relación con la calidad de vida, el dolor y la satisfacción personal en los pacientes sometidos a descompresión neural lumbar aislada en comparación con aquellos que tuvieron la fusión asociada, utilizando como criterios indicativos para artrodesis la presencia de deformidades y / o inestabilidad segmentaria.

Descriptores: Columna vertebral; Dolor de la región lumbar; Artrodesis; Descompresión quirúrgica; Calidad de vida; Ciática; Discectomía.

1. Mestre, Chefe do Grupo de Coluna Vertebral do Departamento de Ortopedia eTraumatologia da Santa Casa da Misericórdia de Santos - Santos, SP, Brasil.

2. Médico Ortopedista, Estagiário do Grupo de Coluna Vertebral do Departamento de Ortopedia eTraumatologia da Santa Casa da Misericórdia de Santos - Santos, SP, Brasil.

3. Doutora, Professora Adjunta do Curso de Fisioterapia da Universidade Federal de São Paulo - São Paulo, SP, Brasil.

Trabalho realizado no Departamento de Ortopedia eTraumatologia - Santa Casa da Misericórdia de Santos - SP. Brasil.

Correspondência: Av. Ana Costa 259 / 51, Encruzilhada - 11060-001 - Santos - SP. Brasil. albertocoluna@yahoo.com.br 


\section{INTRODUÇÃO}

As afecções degenerativas da coluna vertebral são responsáveis por um crescente número de atendimentos nos consultórios médicos ortopédicos e pronto-socorros brasileiros. Estima-se que $77 \%$ da população irá apresentar ao menos um episódio de lombalgia incapacitante em algum momento da vida e 35\% apresentarão dor irradiada no trajeto do nervo ciático ${ }^{1}$. Dentre as alterações degenerativas da coluna lombossacra, a hérnia discal é o diagnóstico mais comum, acometendo 2 a 3\% da população ${ }^{2,3}$. Este é também o principal motivo da realização de cirurgia da coluna vertebral em adultos ${ }^{2}$. Apesar do tratamento conservador ter uma alta taxa de bons resultados ${ }^{4}$ e ser predominante nos casos de dor ciática secundária a hérnias discais ou estenose do canal vertebral, o tratamento cirúrgico pode estar indicado quando não houver regressão do quadro álgico por pelo menos 6 semanas, se persistência e/ou progressão do déficit neurológico e nos casos de crises repetitivas e incapacitantes de lombociatalgia ${ }^{4}$. O conceito fundamental no tratamento cirúrgico destas afecções é a descompressão neural, através de discectomias, foraminotomias ou laminectomias, de acordo com a região anatômica que se apresentar estenótica e sintomática. Entretanto, em algumas situações, pode existir a necessidade de procedimentos estabilizadores da coluna vertebral. A associação da fusão intervertebral está indicada nos pacientes que apresentam deformidades na coluna, levando ao desequilíbrio nos planos coronal ou sagital ${ }^{5}$. Além disto, radiografias lombossacras laterais dinâmicas em flexão e extensão podem determinar a presença de instabilidade segmentar não observadas nas incidências laterais convencionais e que também consistem em uma indicação para a artrodese ${ }^{5}$. Apesar de existirem numerosas publicações relacionadas aos resultados operatórios de afecções degenerativas lombossacras, persiste a controvérsia sobre o impacto da fusão lombar na qualidade de vida. Este estudo teve por objetivo comparar a qualidade de vida, a dor e a satisfação pessoal de pacientes submetidos à descompressão neural lombar isolada àqueles submetidos à descompressão associada à fusão lombossacra, utilizando como critério para a artrodese a presença de deformidades e/ou instabilidade mecânica segmentar.

\section{MATERIAIS E MÉTODOS}

Trata-se de um estudo transversal comparativo. Após aprovação no Comitê de ética em Pesquisa com Seres Humanos (número $52 / 10$ ), os pacientes que preencheram os critérios de inclusão foram convidados a participar desta pesquisa e assinaram o Termo de Consentimento Livre e Esclarecido. Participaram do estudo 44 indivíduos com diagnóstico de hérnia de disco e/ou estenose degenerativa central ou foraminal da coluna lombossacra (incluindo a primeira vértebra lombar ao sacro) que foram submetidos ao tratamento operatório no período compreendido entre março de 2007 e novembro de 2009. O tempo mínimo pós-operatório foi doze meses para ambos os grupos. Todas as cirurgias foram realizadas na Santa Casa da Misericórdia de Santos pelo mesmo cirurgião.

\section{Os pacientes foram divididos em dois grupos:}

a) Grupo "Descompressão" (D): composto por 22 pacientes submetidos à descompressão neural através de discectomia microcirúrgica e/ou foraminotomias, auxiliadas por magnificação de imagens com lupa de 2,5 vezes de aumento (Designs for Vision, Inc., NY, US); b) Grupo "Descompressão e Fusão" (DF): composto por 22 pacientes submetidos à descompressão neural conforme descrição anterior ou através de laminectomia e facetectomias, seguidas de fusão póstero lateral com a utilização de parafusos pediculares. Nestes casos foi utilizado enxertia autóloga, proveniente dos elementos posteriores locais (processos espinhosos, lâmina e facetas articulares), associados a fragmentos ósseos da crista ilíaca posterior direita. As indicações cirúrgicas seguiram os critérios descritos nos Quadros 1 e 2. Consideramos instabilidade mecânica a presença de desequilíbrio do tronco no plano coronal e/ou sagital ou espondilolisteses e retrolisteses vistas nas radiografias laterais simples. Além disto, realizamos radiografias laterais ortostáticas em flexão e extensão máximas, com a finalidade de detectarmos
Quadro 1. Critérios de inclusão e indicações cirúrgicas para descompressão neural lombar isolada.

Pacientes de ambos os sexos

Idade entre 18 e 70 anos

Queixa de dor irradiada no trajeto do nervo ciático uni ou bilateralmente

Diagnóstico de hérnia discal ou estenose degenerativa da coluna lombossacra, central ou foraminal, confirmado por radiografias simples, dinâmicas e ressonância nuclear magnética

Falha do tratamento clínico por, no mínimo, 12 semanas;

Presença de déficit neurológico

Ausência de deformidades ou instabilidade mecânica que necessitasse de fusão intervertebral

Quadro 2. Critérios de inclusão e indicações cirúrgicas para descompressão neural lombar associada a artrodese lombossacra.

\begin{tabular}{|l|}
\hline Pacientes de ambos os sexos \\
\hline Idade entre 18 e 70 anos \\
\hline Queixa de dor irradiada no trajeto do nervo ciático uni ou bilateralmente \\
\hline $\begin{array}{l}\text { Diagnóstico de hérnia discal ou estenose degenerativa da coluna lombossacra, central ou } \\
\text { foraminal, confirmado por radiografias simples, dinâmicas e ressonância nuclear magnética }\end{array}$ \\
\hline $\begin{array}{l}\text { Falha do tratamento clínico por, no mínimo, } 12 \text { semanas; } \\
\text { Presença de déficit neurológico }\end{array}$ \\
\hline $\begin{array}{l}\text { Presença de deformidades ou instabilidade segmentar lombossacra documentada por radio- } \\
\text { grafias laterais simples e dinâmicas em flexão e extensão (angulação maior que } 10 \text { graus ou } \\
\text { translação superior a } 4 \text { milímetros entre } 2 \text { vértebras adjacentes); ou necessidade de descom- } \\
\text { pressão ampla que resultasse em instabilidade iatrogênica }\end{array}$ \\
\hline
\end{tabular}

instabilidade segmentar dinâmica nos níveis a serem descomprimidos. Utilizamos os critérios propostos Herkowitz ${ }^{6}$ e Muggleton et $a l .{ }^{7}$, que incluem a presença de translação igual ou superior a $4 \mathrm{~mm}$ ou ângulação maior ou igual a 10 graus.

Os pacientes foram avaliados clínica e radiograficamente durante as consultas periódicas pós-operatórias. Foram preenchidos questionários referentes ao quadro clínico, uso de medicamentos analgésicos e satisfação pessoal. Além disto, utilizamos a escala visual da dor lombar e ciática. Finalmente, os pacientes responderam ao questionário Oswestry ${ }^{8}$, que avalia a incapacidade funcional devido à dor e o SF-36 ${ }^{9}$, destinado à avaliação da qualidade de vida.

Os dados foram analisados com o programa estatístico SPSS versão 17.0 (SPSS Inc. Chicago, IL, USA).

A análise descritiva é mostrada em média e desvio padrão, mínimos e máximos e porcentagens. As variáveis foram testadas quanto a sua normalidade pelo teste Kolmogorov-Smirnov. Foi aplicado o teste $t$ para amostras independentes para verificar as diferenças entre os grupos D e DF e nas variáveis que apresentaram distribuição normal. Para as variáveis que não apresentaram distribuição normal (VAS ciática, uso de medicamentos, opção de tratamento e domínio "Aspectos Físicos" do SF-36) foi utilizado o teste Mann-Whitney para verificar a diferença entre os grupos. A relação entre a intensidade de dor (lombar e ciática) com o domínio "Dor" do SF-36, com o questionário Oswestry foi verificada por meio do coeficiente de correlação de Pearson e da intensidade de dor com o uso de medicamentos pelo coeficiente de correlação de Spearman. Para todas as análises foi usado nível de significância de 0,05.

\section{RESULTADOS}

O diagnóstico de hérnia discal foi o mais frequentemente encontrado (77\% dos casos) no Grupo D. No grupo DF os diagnósticos mais predominantes foram espôndilo/retrolistese associada a estenose degenerativa (46\%) e hérnia discal associada a instabilidade segmentar (23\%). Em relação ao gênero, em ambos os grupos houve predomínio de homens ( $55 \%$ no grupo D e $75 \%$ no grupo DF).

$\mathrm{Na}$ Tabela 1 podem ser observados os resultados descritivos das variáveis analisadas no estudo: tempo de pós-operatório (meses), idade (anos), intensidade da dor lombar e ciática, SF-36 (domínios "Dor", "Capacidade Funcional" e "Aspectos Físicos") e questionário Oswestry. 
Não houve diferença estatisticamente significativa entre os grupos D e DF para as seguintes variáveis analisadas: tempo de pósoperatório $(p=0,974)$, idade $(p=0,110)$, intensidade da dor lombar $(p=0,890)$, intensidade da dor ciática $(p=0,314)$, domínios do SF36 ("dor", $p=0,688$; "capacidade funcional", $p=0,981$; "aspectos físicos", $p=0,956)$, questionário Oswestry $(p=0,408)$.

A Figura 1 mostra a distribuição da amostra de acordo com as análises dos domínios do questionário SF-36, do questionário Oswestry e das intensidades de dor lombar e ciática.

Ambos os grupos apresentaram bons níveis de qualidade de vida e pouco impacto da dor lombar e/ou ciática nas atividades diárias. Para os domínios "Dor" e "Capacidade Funcional" os resultados foram melhores, sendo que mais de $50 \%$ dos grupos apresentaram valores maiores do que 50, que indica um índice bom de qualidade de vida. No entanto, para o domínio "Aspectos Físicos" menos de $40 \%$ dos dois grupos apresentaram estes resultados.

Para o impacto da dor na vida do indivíduo nota-se que $75 \%$ do Grupo D e 60\% do Grupo DF apresentaram índices de até 30\%, o que representa baixo impacto da dor nas atividades diárias.

Na Figura 2 pode-se observar a distribuição dos indivíduos de acordo com as intensidades de dor lombar e ciática avaliadas pelas escalas numéricas. Apresenta-se também a classificação qualitativa desta distribuição de acordo com Breivik et al. ${ }^{10}$.

Tabela 1. Análise descritiva da amostra estudada.

\begin{tabular}{c|c|c|c|c|c|c}
\hline & \multicolumn{3}{|c|}{ Grupo D } & \multicolumn{3}{c}{ Grupo DF } \\
\cline { 2 - 7 } & Média (DP) & Mínimo & Máximo & Média (DP) & Mínimo & Máximo \\
\hline Tempo de PO & $22(9)$ & 12 & 44 & $22(7)$ & 12 & 32 \\
\hline Idade & $64(16)$ & 28 & 87 & $57(12)$ & 43 & 80 \\
\hline Intensidade Dor Lombar & $3(3)$ & 0 & 8 & $3(3)$ & 0 & 9 \\
\hline Intensidade Dor Ciática & $2(3)$ & 0 & 10 & $1(2)$ & 0 & 6 \\
\hline Dor & $56(23)$ & 20 & 100 & $59(25)$ & 31 & 100 \\
\hline Capacidade Funcional & $64(26)$ & 10 & 100 & $64(24)$ & 10 & 100 \\
\hline Aspectos Físicos & $44(44)$ & 0 & 100 & $43(47)$ & 0 & 100 \\
\hline Oswestry & $19(16)$ & 0 & 46 & $23(17)$ & 0 & 46 \\
\hline
\end{tabular}

(A)

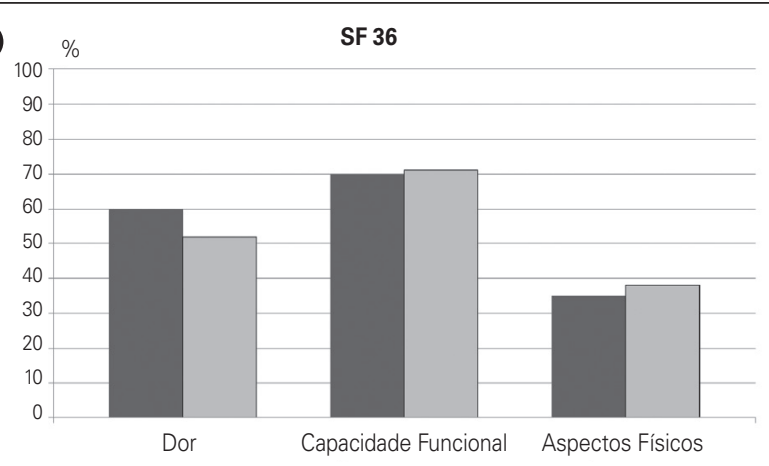

B

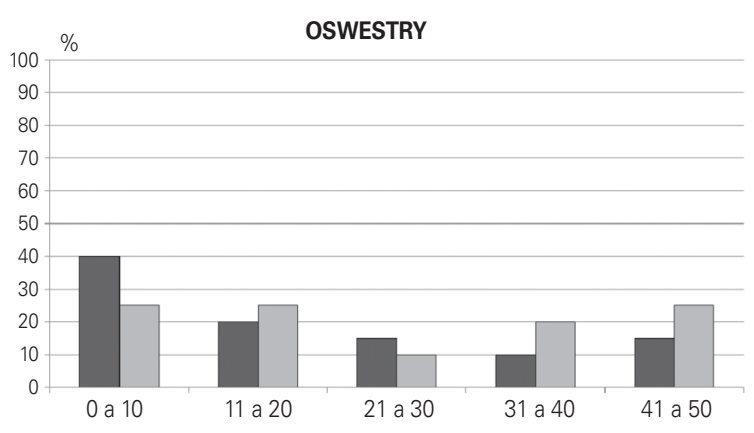

índice final do questionário Oswestry (\%)

Grupo D Grupo DF

Figura 1. (A) Observa-se os resultados dos domínios do questionário SF-36 (Valores acima do índice 50 em cada domínio). (B) Resultados do questionário Oswestry.
Em relação à intensidade da dor lombar pode-se observar que aproximadamente 20 e 30\% dos indivíduos dos Grupos D e DF, respectivamente, não relataram nenhuma dor (índice zero no gráfico), porém a maior parte (55\%) do Grupo D relatou dor lombar variando de leve a moderada. Para a intensidade da dor ciática 50\% do Grupo D e 61\% do Grupo DF referiu não sentir nenhuma dor, sendo que o restante dos indivíduos do Grupo DF apresentou dor ciática de suave a moderada, não referindo dor severa como alguns representantes (15\%) do Grupo D.

$\mathrm{Na}$ Tabela 2 são apresentados os resultados de distribuição da amostra em relação ao uso de medicamentos e opção pelo tratamento cirúrgico.

Nota-se que $100 \%$ do grupo D e $90 \%$ do grupo DF aceitaria a cirurgia que foi realizada caso ainda não tivesse operado e que a maior parte dos pacientes de ambos os grupos não faz uso de medicação atualmente. Também não foi encontrada diferença estatisticamente significativa entre os grupos para o uso de medicamentos $(p=0,805)$ e a opção de tratamento cirúrgico $(p=0,647)$.

As análises de correlação entre as várias formas de avaliação da dor são mostradas na Tabela 3.

\section{A}

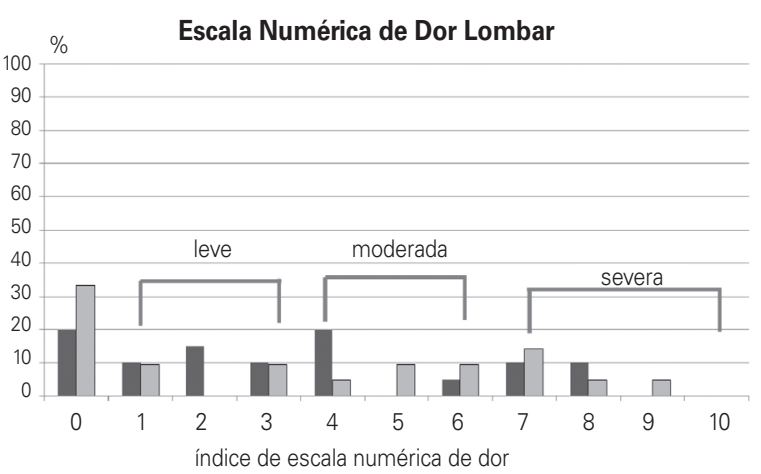

B

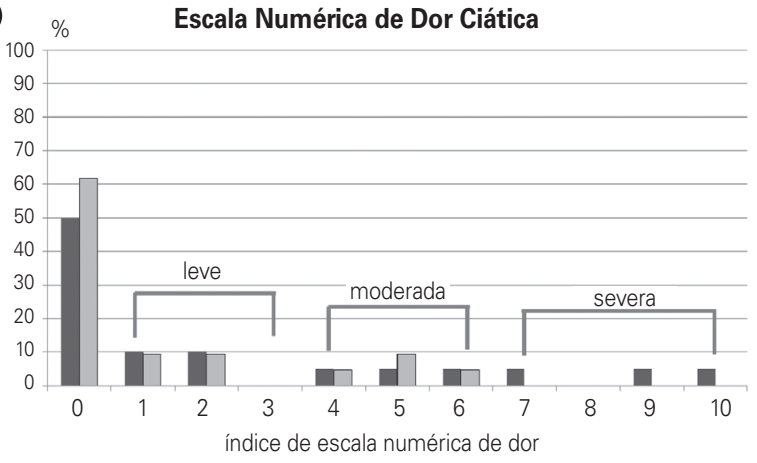

Grupo D

$\square$ Grupo DF

Figura 2. (A) Resultados da escala numérica de dor lombar. (B) Resultados da escala numérica de dor ciática. Classificação qualitativa baseada em Breivik et al. ${ }^{10}$.

Tabela 2. Distribuição por porcentagem da amostra de acordo com gênero, o uso de medicamentos e opção de tratamento.

\begin{tabular}{|c|c|c|c|}
\hline & & Grupo D & Grupo DF \\
\hline \multirow{5}{*}{$\begin{array}{l}\text { "Qual das seguintes } \\
\text { respostas melhor des- } \\
\text { creve a quantidade e } \\
\text { os medicamentos que } \\
\text { toma por causa das } \\
\text { suas costas?" }\end{array}$} & "Nenhum" & $50 \%$ & $61 \%$ \\
\hline & \begin{tabular}{|c} 
"Não-narcótico uma vez por semana \\
ou menos" \\
\end{tabular} & $40 \%$ & $14 \%$ \\
\hline & "Não-narcótico diariamente" & $5 \%$ & $10 \%$ \\
\hline & "Narcótico semanalmente ou menos" & $0 \%$ & $10 \%$ \\
\hline & "Narcótico diariamente" & $5 \%$ & $5 \%$ \\
\hline \multirow{5}{*}{$\begin{array}{l}\text { "Se você ainda não } \\
\text { tivesse operado, acei- } \\
\text { taria a cirurgia que foi } \\
\text { realizada?" }\end{array}$} & "Com certeza Sim" & $85 \%$ & $80 \%$ \\
\hline & "Provavelmente Sim" & $15 \%$ & $10 \%$ \\
\hline & "Não sei" & $0 \%$ & $0 \%$ \\
\hline & "Provavelmente Não" & $0 \%$ & $0 \%$ \\
\hline & "Com certeza Não" & $0 \%$ & $10 \%$ \\
\hline
\end{tabular}


Tabela 3. Análises de correlação da intensidade de dor lombar e dor ciática com outras variáveis de dor do estudo.

\begin{tabular}{c|c|c|c|c}
\hline & \multicolumn{2}{|c|}{ Grupo D } & \multicolumn{2}{c}{ Grupo DF } \\
\hline & $\mathrm{R}$ & $\mathrm{p}$ & $\mathrm{r}$ & $\mathrm{P}$ \\
\hline $\begin{array}{c}\text { Intensidade dor lombar com } \\
\text { domínio dor SF36 }\end{array}$ & $-0,46$ & $0.040^{*}$ & $-0,53$ & $0.013^{*}$ \\
\hline Oswestry & 0,80 & $0.000^{*}$ & 0,47 & $0.032^{*}$ \\
\hline Uso de medicamentos & 0,48 & $0.033^{*}$ & 0,59 & $0.005^{*}$ \\
\hline $\begin{array}{c}\text { Intensidade dor ciática com } \\
\text { domínio de dor SF36 }\end{array}$ & $-0,43$ & $0.059^{*}$ & $-0,47$ & $0.033^{*}$ \\
\hline Oswestry & 0,75 & $0.000^{*}$ & 0,37 & 0.100 \\
\hline Uso de medicamentos & 0,19 & 0.431 & 0,25 & 0.266 \\
\hline${ }^{*}$ correlacão estatisticamente significativa & & & &
\end{tabular}

${ }^{*}$ correlação estatisticamente significativa a $p<0,05$.

No Grupo D foi encontrada correlação estatisticamente significativa entre a intensidade da dor Lombar e o questionário Oswestry (alta correlação), o domínio "Dor" do SF-36 (baixa correlação), e o uso de medicamentos (baixa correlação). Para a intensidade de dor Ciática, as correlações foram estatisticamente significativas com o Oswestry (correlação alta) e com o Domínio "Dor" do SF-36 (baixa correlação) e Apenas a correlação entre a intensidade da dor Ciática e o uso de medicamentos não foi significante.

Já para o Grupo DF as correlações da intensidade de dor Lombar foram todas estatisticamente significativas, variando de baixa a moderada. Para a intensidade de dor Ciática não houve correlação estatisticamente significativa com o uso de medicamentos ou com o questionário Oswestry.

Dois pacientes do grupo 1 foram excluídos por necessitarem de re-intervenção cirúrgica e fusão devido à recidiva da doença. De maneira análoga, um paciente do grupo 2 apresentou a "nã̃o-consolidação" e a falha tardia da instrumentação (soltura do implante) e necessitou de revisão operatória.

\section{DISCUSSÃO}

A avaliação funcional de pacientes com diagnóstico de artrodese lombossacra é uma tema de grande interesse entre os cirurgiões de coluna. Persiste o questionamento sobre o real impacto da fusão na qualidade de vida desta população e se estes pacientes apresentariam pior desempenho físico quando comparados àqueles submetidos à descompressão neural isolada.

Apesar de concordarmos que a melhor maneira de avaliarmos esta e outras questões clínicas seria através de estudos prospectivos e randomizados, por apresentarem o maior nível de evidência científica ${ }^{11}$, acreditamos que, nesta situação, tal desenho de estudo não se aplicaria, uma vez que as indicações cirúrgicas para a descompressão simples ou associada a fusão são distintas. Assim, encontramos na avaliação transversal, uma maneira viável de compararmos os participantes.

Os grupos eram homogêneos em relação a idade, gênero e tempo de pós-operatório $(p>0,05)$. Para avaliarmos a qualidade de vida, utilizamos os questionários SF-36 e Oswestry, validados para a língua portuguesa ${ }^{8,9}$ Ambos forneceram informações importantes em relação à dor, capacidade funcional e aspectos físicos, além de avaliarem o impacto da dor nas atividades diárias destes indivíduos. Utilizamos também a Escala Numérica de Dor lombar e ciática, tornando nossa metodologia multidimensional, característica importante na avaliação de enfermidades crônicas ${ }^{10}$.

\section{REFERÊNCIAS}

1. Gotfryd A, Avanzi O. A systematic review of randomised clinical trials using posterior discectomy to treat lumbar disc herniations. Int Orthop. 2009:33(1):11-7.

2. Vialle LR, Vialle EN, Henao JES, Giraldo G. Hérnia discal lombar. Rev Bras Ortop. 2010; 45(1): 17-22.

3. Ortiz J, Abreu AD. Tratamento cirúrgico das hérnias discais lombares em regime ambulatorial. Rev Bras Ortop. 2000; 35(11/12):440-6.

4. Hennemann SA, Schumacher W. Hérnia de disco lombar: revisão de conceitos atuais. Rev Bras Ortop. 1994; 29(3): 115-126.

5. Resnick DK, Choudhri TF, Dailey AT, Groff MW, Khoo L, Matz PG, et al.Guidelines for the performance of fusion procedures for degenerative disease of the lumbar spine. Part 8 : lumbar fusion for disc herniation and radiculopathy. J Neurosurg Spine.2005:2(6):673-8.

6. Herkowitz HN. Spine update. Degenerative lumbar spondylolisthesis. Spine (Phila Pa 1976). 1995;20(9):1084-90.

7. Muggleton JM, Kondracki M, Allen R. Spinal fusion for lumbar instability: does it have a scientific basis? J Spinal Disord. 2000;13(3):200-4

8. Vigatto R, Alexandre NM, Correa Filho HR. Development of a Brazilian Portuguese version of the Oswestry Disability Index: cross-cultural adaptation, reliability, and validity. Spine (Phila Pa 1976). 2007;32(4):481-6.

9. Ciconelli RM, Ferraz MB, Santos W, Meinão I, Quaresma MR. Tradução para a língua portuguesa e validação do questionário genérico de avaliação de qualidade de vida SF-36 (Brasil SF-36). Rev Bras Reumatol. 1999: 39:143-50.
Os níveis de qualidade de vida avaliados por meio dos domínios "Dor" e "Capacidade Funcional" do questionário SF-36 apresentaram bons resultados. Já para o domínio "Aspectos Físicos", os resultados foram regulares. Nossos resultados foram semelhantes aos de Sun et al. ${ }^{12}$, em acompanhamento pós-operatório de 2 anos de pacientes submetidos à discectomia simples, que encontraram boa qualidade de vida nos domínios "Capacidade Funcional" e "Aspectos Físicos". Throckmorton et al. ${ }^{13}$ encontrou resultados semelhantes aos nossos para os domínios "Dor", "Capacidade Funcional" e "Aspectos Físicos" do SF-36, ao avaliar pacientes submetidos à descompressão neural associada à fusão em dois anos de seguimento, demonstrando boa qualidade de vida após a intervenção cirúrgica.

De forma geral, a qualidade de vida foi semelhante nos pacientes submetidos ou não a fusão. Pode-se assim presumir que a artrodese, em desacordo com outros autores ${ }^{3,5,14}$, não aumentou a incapacidade funcional e a limitação física destes pacientes.

O questionário Oswestry fornece dados sobre o impacto da dor lombar e/ou ciática nas atividades diárias. Neste estudo obtivemos excelentes resultados neste questionário para os dois grupos, mostrando pouco impacto da dor e boa capacidade funcional. Ohtori et al. ${ }^{15}$ submeteram 45 pacientes à discectomia simples e encontraram resultados semelhantes aos nossos para o Oswestry e a intensidade da dor ciática, porém resultados inferiores para a intensidade de dor lombar. Já Glassman et al. ${ }^{16}$, em estudo com pacientes submetidos à descompressão neural e fusão, encontraram resultados semelhantes aos nossos para o Oswestry e a intensidade da dor lombar, porém resultados maiores para a intensidade da dor ciática.

Os valores para a intensidade da dor ciática residual foram baixos nos dois grupos, evidenciando que o objetivo principal (descompressão neural) foi atingido e compatível com publicações existentes ${ }^{15,17}$ Encontramos baixos níveis de dor lombar em ambos os grupos. Apesar da redução da dor lombar não ser um desfecho primário destas cirurgias, o mesmo foi alcançado e considerado como satisfatório.

Foram realizadas as análises de correlação entre a intensidade de dor lombar e ciática com o domínio "Dor" do SF-36, o questionário Oswestry e o uso de medicamentos, para confirmação dos níveis de qualidade de vida e dos aspectos clínicos da amostra estudada.

Para a intensidade de dor lombar todas as correlações foram estatisticamente significativas nos dois grupos, sendo mais fortes no Grupo $D$, sugerindo que os níveis de qualidade de vida são bons, em curto prazo, tanto em pacientes submetidos à descompressão neural quanto naqueles submetidos à fusão associada. O mesmo foi observado por Eie et al. ${ }^{18}$ que reportaram $89 \%$ de bons resultados clínicos no período pós-operatório precoce de pacientes submetidos, ou não, à fusão.

A principal limitação do presente estudo foi o reduzido período de seguimento, nos impossibilitando concluirmos sobre o papel tardio da fusão, da recidiva dos sintomas ou da presença de degeneração discal nos níveis vertebrais adjacentes.

\section{CONCLUSÃO}

Não encontramos diferenças em relação à qualidade de vida, à dor e à satisfação pessoal em pacientes submetidos à descompressão neural lombar isolada àqueles que tiveram a fusão associada, utilizando como critério indicativo para artrodese a presença de deformidades e/ou instabilidade segmentar.

10. Breivik H, Borchgrevink PC, Allen SM, Rosseland LA, Romundstad L, Hals EK, Kvarstein G, et al. Assessment of pain. Br J Anaesth. 2008:101(1):17-24.

11. Cook DJ, Guyatt GH, Laupacis A, Sackett DL. Rules of evidence and clinical recommendations on the use of antithrombotic agents. Chest. 1992;102(4Suppl):305S-311S.

12. Sun EC, Wang JC, Endow K, Delamarter RB. Adjacent two-level lumbar discectomy: outcome and SF-36 functional assessment. Spine (Phila Pa 1976). 2004:29(2):E22-7.

13. Throckmorton TW, Hilibrand AS, Mencio GA, Hodge A, Spengler DM. The impact of adjacent level disc degeneration on health status outcomes following lumbar fusion. Spine (Phila Pa 1976). 2003;28(22):2546-50.

14. Donceel P, Du Bois M. Fitness for work after surgery for lumbar disc herniation: a retrospective study. Eur Spine J. 1998;7(1):29-35.

15 Ohtori S Yamashita M Yamauchi K, Inoue G, KoshiT Suzuki M et al. Low back pain after lumbar discectomy in patients showing endplate modic type 1 change. Spine (Phila Pa 1976). 2010;35(13):E596-600.

16. Glassman SD, Carreon LY, Djurasovic M, Dimar JR, Johnson JR, Puno RM, Campbell MJ. Lumbar fusion outcomes stratified by specific diagnostic indication. Spine J. 2009:9(1):13-21.

17. Häkkinen A, Kautiainen $H$, Järvenpää S, Arkela-Kautiainen $M$, Ylinen J. Changes in the total Oswestry Index and its ten items in females and males pre- and post-surgery for lumbar disc herniation: a 1-year follow-up. Eur Spine J. 2007;16(3):347-52.

18. Eie N. Comparison of the results in patients operated upon for ruptured lumbar discs with and without spinal fusion. Acta Neurochir (Wien).1978;41(1-3):107-13. 